\title{
BMJ Open Effect of physical exercise cessation on strength, functional, metabolic and structural outcomes in older adults: a protocol for systematic review and meta- analysis
}

\author{
Ángel Buendía-Romero (D) , ${ }^{1}$ Tomas Vetrovsky, ${ }^{2}$ Fernando Estévez-López (i) , ${ }^{3}$ \\ Javier Courel-lbáñez (ib) ${ }^{1,2}$
}

To cite: Buendía-Romero Á, Vetrovsky T, Estévez-López F, et al. Effect of physical exercise cessation on strength, functional, metabolic and structural outcomes in older adults: a protocol for systematic review and meta-analysis. BMJ Open 2021;11:e052913. doi:10.1136/ bmjopen-2021-052913

- Prepublication history and additional supplemental material for this paper are available online. To view these files, please visit the journal online (http://dx.doi.org/10.1136/ bmjopen-2021-052913).

Received 30 April 2021 Accepted 12 November 2021

Check for updates

(C) Author(s) (or their employer(s)) 2021. Re-use permitted under CC BY-NC. No commercial re-use. See rights and permissions. Published by BMJ.

${ }^{1}$ Human Performance and Sports Science Laboratory, Faculty of Sport Sciences, University of Murcia, Murcia, Spain

${ }^{2}$ Faculty of Physical Education and Sport, Charles University, Praha, Czech Republic ${ }^{3}$ Department of Child and Adolescent Psychiatry, Erasmus MC, Rotterdam, The Netherlands

\section{Correspondence to} Dr Fernando Estévez-López; fer@estevez-lopez.com and Dr Javier Courel-Ibáñez; javier.courel.ibanez@gmail.com

\section{ABSTRACT}

Introduction There is not a doubt that tailored exercise is an effective non-pharmacological approach for preventing, mitigating and even reversing ageing-related alterations. However, older adults are likely to experience prolonged periods of inactivity and training cessation periods as a consequence of falls or hospitalisation. Although recent evidence supports that exercise could have a protective effect and help in recovering, there is to date a lack of consensus about what kind of physical exercise prescription and training duration would produce better outcomes after training cessation periods. The current study will determine the effects that available exercise prescriptions produced in older adults in preserving physical conditioning following inactivity periods.

Methods and analysis A systematic search of the literature will be conducted in three databases, namely PubMed, Scopus and Web of Science, from inception to 1 February 2021. Only randomised controlled trials written in English or Spanish will be eligible. No year of publication restriction will be applied. Eligible studies will contain information on population (older adults over 60 years old), intervention (inactivity period, exercise programme their duration), comparator (treatment as usual or waiting list) and outcomes (strength, functional capacity, metabolic health and skeletal muscle structure). Two independent reviewers will (1) search, screen and select studies, (2) extract data about their main characteristics and (3) evaluate their methodological and reporting quality. When disagreements emerge, the reviewers will discuss to reach a consensus. We plan to conduct meta-analysis to quantitatively synthesise the effects under study. Ethics and dissemination As systematic reviews use publicly available data, no formal ethical review and approval are needed. Findings will be published in a peerreviewed journal(s) and presented at conferences. PROSPERO registration number CRD42021235092.

\section{INTRODUCTION}

The population over the age of 60 is increasing day by day. ${ }^{1}$ Ageing could be associated with a progressive health decline, but
Strengths and limitations of this study

- This study will be conducted in accordance with the Preferred Reporting Items for Systematic Reviews and Meta-Analyses.

- We will include randomised controlled trials in which older adults received an exercise prescription of any kind (resistance training, endurance training, concurrent training, multicomponent training) before a training cessation period of any duration (days, weeks or months).

- To assess the effect of training cessation, standardised mean differences (Hedges' g) between the exercise and control groups will be calculated for each outcome and entered into random-effect meta-analysis.

- If applicable, subgroup analyses will be conducted to exclude differences related to age groups or frailty condition.

- The inclusion of heterogeneous samples and a large variety of exercise interventions may be a limitation.

there is not a doubt that tailored exercise ${ }^{2}$ is an effective non-pharmacological approach for preventing ageing-related syndromes (ie, frailty, sarcopenia and functional disability). ${ }^{34}$ Regular physical activity produces cardiovascular, hormonal and skeletal muscle adaptations that counteract muscle atrophy and loss of bone density and contribute to increases in peak oxygen consumption and improvements of mitochondrial biogenesis. ${ }^{5}$ Besides, short-term (1-4 weeks) benefits of exercise programmes have been recently reported in vulnerable populations such as institutionalised older adults. ${ }^{6-9}$

Different modalities of physical exercise programmes have been shown to produce diverse physical outcomes in older adults. Traditionally, resistance training (exercises against an external load to improve 
strength) and endurance training (continuous aerobic/ anaerobic exercise to improve cardiopulmonary fitness) have been prescribed separately for enhancing particular outcomes. ${ }^{10-12}$ Advances in training methods lead to combined programmes such as concurrent training (combination of resistance and endurance training within the same session) or high-intensity intermittent training (repeated bouts of exercise at an intense effort interspersed by low-intensity exercise or periods of rest with varied recovery times), with promising results in health-related outcomes (eg, functionality, strength, body composition, resting heart rate and blood pressure) and greater adherence as a result of time saving. ${ }^{13-16}$ Besides, supervised multicomponent programmes (combination of strength, balance, agility and cardiovascular exercises) has been suggested as the preferred strategy to mitigate ageing-related complications. ${ }^{9}{ }^{17-19}$ In addition to the particularities among training disciplines, new trends in sport sciences appeal to more individualised regimens with a variety of exercises, stimuli and intensities which are controlled by incorporating new technologies and monitoring tools. ${ }^{20-25}$

When people stop exercising regularly or there is an insufficient training stimulus, they may suffer a partial or complete loss of training-induced adaptations, namely the detraining effect. ${ }^{26}$ While older adults commonly suffer from hospitalisation, illness or immobilisation that temporarily disrupt any physical activity, ${ }^{27}$ prior studies have demonstrated that benefits of exercise may persist after short-term ${ }^{28} 29$ and even long-term ${ }^{30-32}$ exercise cessation in community-dwelling. However, other studies examining institutionalised older adults have reported inconclusive results on protective effect. ${ }^{6} 93$ Thus, although supervised multicomponent exercise is considered one of the most effective treatments against ageing-related physical syndromes, ${ }^{3}{ }^{34}$ there is a lack of consensus on what kind of physical exercise prescription and training duration produce better protective effects after exercise cessation in both community-dwelling and institutionalised older adults.

\section{Objective}

This study aims to conduct a systematic review and metaanalysis to determine the effects of available exercise prescriptions in older adults in preserving physical conditioning after inactivity periods.

\section{Review question}

What kind of physical exercise prescription produces better residual/protective effects on strength, functional, metabolic and structural health in the short term and long term in older adults?

\section{METHODS}

\section{Inclusion criteria}

According to the PICOS approach, the inclusion criteria will be:
1. Participants: people aged 60 years and over who have completed a physical training programme followed by an exercise cessation phase.

2. Intervention: exercise training programmes (home based or supervised) of any type (resistance training, endurance training, concurrent training, multicomponent training) immediately followed by a training cessation period. Considering this review aims to identify what kind of physical exercise prescription produces better residual/protective effects, no duration restriction will be set for either the exercise or the cessation interventions to avoid potential bias. Training cessation phase will be considered as any follow-up measures with no active intervention (eg, forced exercise inactivity, hospitalisation, usual daily activity).

3. Comparator: a control group including participants who continued with their usual lifestyle and did not perform physical exercise.

4. Outcome measures: functional capacity measured by validated batteries (eg, Senior Fitness Test, Short Physical Performance Battery, Fullerton Fitness Test) or separate tests (eg, sit-to-stand, timed up and go, foot up and go, balance tests); maximal or submaximal, upperlimb or lower-limb strength measured by dynamic, isometric or isokinetic tests; maximal or submaximal, cardiovascular performance measured by validated aerobic tests with indirect calorimetry, lactate, of estimated equations methods; walking/gait speed; metabolic profile measured by tensiometer or/and blood analysis; body composition and skeletal muscle structure measured by bioimpedance, biopsy, MR or ultrasounds scan.

5. Studies: randomised controlled trials written in English or Spanish that should have at least (1) a control group (eg, treatment as usual or waiting list) and (2) an intervention group (exercise training interventions followed by a training cessation period). Studies with a control group and several intervention groups will also be considered.

No date restrictions will be applied.

\section{Exclusion criteria}

1. Studies without primary data (eg, reviews).

2. Works published in journals without peer-review.

3. Educational and behavioural interventions will be excluded.

\section{Search strategy for identifying relevant studies}

Two independent reviewers (ÁB-R and JC-I) will perform an electronic search in PubMed, MEDLINE and Web of Science Core Collection on 1 February 2021. The primary systematic literature search strategy will include the terms elder, elderly, older adults, detraining, training cessation, exercise interruption, deconditioning, retraining and physical restraint (online supplemental material 1). 


\section{Selection of studies for inclusion in the review}

Metadata will be imported into the Mendeley Desktop, where duplicates will be automatically deleted. A data extraction sheet will be developed. Eligibility assessment will be performed by two reviewers independently (ÁB-R and JC-I) . Relevant review articles will be screen for potential studies. ${ }^{8536}$ First, the studies will be screened by title and abstract. Second, the selected studies will be examined in full text to decide their definitive inclusion. When disagreements emerge between the two independent researchers, consensus will be obtained through discussion, or when required, the opinion of a third researcher (TV) will be considered.

\section{Assessment of methodological quality and reporting of data}

The Grading of Recommendations Assessment, Development and Evaluation framework will be used to assess the quality of the evidence across studies for each outcome separately. Risk of bias (RoB) 2 tool will be used to assess the RoB in randomised trials included in Cochrane Reviews. Studies with a score of at least five points will be considered as having high RoB. Two researchers will independently assess the RoB and the inconsistency, indirectness, imprecision and publication bias (ÁB-R and JC-I) of each eligible study. Disagreements on these assessments will be solved in a consensus meeting between the independent reviewers with another member of the team (TV).

\section{Data extraction and management}

Two independent reviewers (ÁB-R and JC-I) will extract the following data for each study: (1) characteristics of trial participants (total sample number, sex, age, weight, height, body composition); (2) details of the exercise intervention (volume, intensity, duration, exercises included, technological resources); (3) details of the training cessation period (type of inactivity, duration); (4) details of the control group (eg, education about nutrition or physical activity); (5) outcome measures (strength, functional, metabolic and structural variables); (6) methodological quality of individual studies, according to the Cochrane Handbook for Systematic Reviews and (7) funding sources. When discrepancies emerge in the coding between the two researchers' results, these will be discussed with a third reviewer (TV) to reach a consensus.

\section{Data synthesis and analysis}

To assess the effect of training cessation, standardised mean differences (Hedges' g) between the exercise and control groups will be calculated for each outcome and entered into random-effect meta-analysis. Effect sizes of $0.2,0.4$ and 0.8 will be considered as small, moderate and large, respectively. To check the robustness of the primary analysis, sensitivity analysis will be carried out by excluding studies with high RoB. Because hospitalisation usually resembles immobilisation, and therefore, the detraining effect will worsen much more than if there is a minimum stimulus such as exercise inactivity or daily activity, we will consider conducting subgroup analyses considering hospitalisation separately if possible. In addition, we will analyse different groups of older adults over 60 years with subgroups analyses considering: (1) different age groups (2) institutionalised or community-dwelling and (3) frailty level or fitness status if possible. Heterogeneity will be assessed using the $\mathrm{I}^{2}$ statistic (the percentage of total variation attributed to between-study heterogeneity). Inconsistency across studies will be considered serious when heterogeneity will be high $\left(\mathrm{I}^{2} \geq 50 \%\right)$. In case of observing high heterogeneity $\left(\mathrm{I}^{2} \geq 50 \%\right)$ and a minimum number of 10 studies, potential effect moderators will be explored with meta-regression models using the metafor package in R. Indirectness will be considered serious when interventions include both exercise and additional components (ie, cointerventions). Imprecision will be considered serious when the $95 \%$ CI will be wide and will cross the line of no effect. Finally, the presence of publication bias and small study effects will be assessed using visual inspection of a funnel plot and randomeffects version of Egger's regression test. All analyses will be performed using metafor package in $\mathrm{R}$ (The R Foundation for Statistical Computing, Vienna, Austria). Anticipated timeline for review: screening of search results by 5 November 2021, data analysis and write-up by 15 April 2022.

\section{Presentation and reporting of results}

The findings of the present systematic review and metaanalysis will be reported following the Preferred Reporting Items for Systematic Reviews and Meta-Analyses statement. ${ }^{37}$ We will illustrate the process of study selection using a flow diagram. A table with the main characteristics of each study will also be provided.

\section{Ethics and dissemination}

As systematic reviews use publicly available data, no formal ethical review and approval are needed. Findings will be published in a peer-reviewed journal(s) and presented at conferences.

\section{Patient and public involvement}

An institutionalised older adult and a nursing home manager as research partners were actively involved in the design of the present systematic review and metaanalysis and will be involved in all the steps described in this protocol. Patients' associations and the general public will be reached through several approaches, including talks and discussions.

\section{Limitations}

The inclusion of heterogeneous samples and a large variety of exercise interventions may be a limitation. The conclusions from this review might have a limited generalisability to healthy adults who completing exercise on a regular basis. There is no guarantee the search was completely exhaustive despite the extensive search strategy. 
Twitter Ángel Buendía-Romero @AngelBuenRome

Contributors ÁB-R, TV, FE-L and JC-I designed the protocol. ÁB-R drafted the manuscript with the senior supervision by FE-L and JC-I. ÁB-R, TV, FE-L and JC-I revised and approved the final version of the manuscript. FE- $L$ and JC-I will be the guarantors of the review.

Funding ÁB-R and JC-I are supported by the Autonomous Community of the Region of Murcia, Regional Programme for the Promotion of Scientific and Technical Research (Action Plan 2018), Seneca Foundation-Agency of Science and Technology, Region of Murcia (ID: 20872/PI/18). FE-L is supported by the European Union's Horizon 2020 research and innovation program under the Marie Skłodowska-Curie (grant agreement no. 707404).

Competing interests None declared.

Patient consent for publication Not applicable.

Provenance and peer review Not commissioned; externally peer reviewed.

Supplemental material This content has been supplied by the author(s). It has not been vetted by BMJ Publishing Group Limited (BMJ) and may not have been peer-reviewed. Any opinions or recommendations discussed are solely those of the author(s) and are not endorsed by BMJ. BMJ disclaims all liability and responsibility arising from any reliance placed on the content. Where the content includes any translated material, BMJ does not warrant the accuracy and reliability of the translations (including but not limited to local regulations, clinical guidelines, terminology, drug names and drug dosages), and is not responsible for any error and/or omissions arising from translation and adaptation or otherwise.

Open access This is an open access article distributed in accordance with the Creative Commons Attribution Non Commercial (CC BY-NC 4.0) license, which permits others to distribute, remix, adapt, build upon this work non-commercially, and license their derivative works on different terms, provided the original work is properly cited, appropriate credit is given, any changes made indicated, and the use is non-commercial. See: http://creativecommons.org/licenses/by-nc/4.0/.

\section{ORCID iDs}

Ángel Buendía-Romero http://orcid.org/0000-0001-7044-8191

Fernando Estévez-López http://orcid.org/0000-0003-2960-4142

Javier Courel-Ibáñez http://orcid.org/0000-0003-2446-1875

\section{REFERENCES}

1 Beard JR, Bloom DE. Towards a comprehensive public health response to population ageing. Lancet 2015;385:658-61.

2 Fragala MS, Cadore EL, Dorgo S, et al. Resistance training for older adults: position statement from the National Strength and Conditioning Association. J Strength Cond Res 2019;33:2019-52.

3 Cadore EL, Casas-Herrero A, Zambom-Ferraresi F, et al. Multicomponent exercises including muscle power training enhance muscle mass, power output, and functional outcomes in institutionalized frail nonagenarians. Age 2014;36:773-85.

4 García-Hermoso A, Ramirez-Vélez R, Sáez de Asteasu ML, et al. Safety and effectiveness of long-term exercise interventions in older adults: a systematic review and meta-analysis of randomized controlled trials. Sports Med 2020;50:1095-106.

5 Valenzuela PL, Castillo-García A, Morales JS, et al. Physical exercise in the oldest old. Compr Physiol 2019;9:1281-304.

6 Courel-Ibáñez J, Pallarés JG, García-Conesa S, et al. Supervised Exercise (Vivifrail) Protects Institutionalized Older Adults Against Severe Functional Decline After 14 Weeks of COVID Confinement. J Am Med Dir Assoc 2021;22:217-9.

7 Buendía-Romero Ángel, García-Conesa S, Pallarés JG, et al. Effects of a 4-week multicomponent exercise program (Vivifrail) on physical frailty and functional disability in older adults living in nursing homes. CPD 2020;20:74-81.

8 Sáez de Asteasu ML, Martínez-Velilla N, Zambom-Ferraresi F, et al. Changes in muscle power after usual care or early structured exercise intervention in acutely hospitalized older adults. J Cachexia Sarcopenia Muscle 2020;11:jcsm.12564

9 Courel-lbáñez J, Buendía-Romero Ángel, Pallarés JG, et al. Impact of tailored multicomponent exercise for prevent weakness and falls on nursing home residents' functional capacity. J Am Med Dir Assoc 2021;385.

10 Murias JM, Kowalchuk JM, Paterson DH. Time course and mechanisms of adaptations in cardiorespiratory fitness with endurance training in older and young men. J Appl Physiol 2010;108:621-7.
11 DiPietro L, Dziura J, Yeckel CW, et al. Exercise and improved insulin sensitivity in older women: evidence of the enduring benefits of higher intensity training. J Appl Physiol 2006;100:142-9.

12 Miszko TA, Cress ME, Slade JM, et al. Effect of strength and power training on physical function in community-dwelling older adults. $J$ Gerontol A Biol Sci Med Sci 2003;58:M171-5.

13 García-Pinillos F, Laredo-Aguilera JA, Muñoz-Jiménez M, et al. Effects of 12-week concurrent high-intensity interval strength and endurance training program on physical performance in healthy older people. J Strength Cond Res 2019;33:1445-52.

14 Cadore EL, Pinto RS, Lhullier FLR, et al. Physiological effects of concurrent training in elderly men. Int J Sports Med 2010;31:689-97.

15 Coetsee $\mathrm{C}$, Terblanche $\mathrm{E}$. The effect of three different exercise training modalities on cognitive and physical function in a healthy older population. Eur Rev Aging Phys Act 2017;14:1-10.

16 Coswig VS, Barbalho M, Raiol R, et al. Effects of high vs moderateintensity intermittent training on functionality, resting heart rate and blood pressure of elderly women. J Transl Med 2020;18:1-11.

17 Izquierdo M, Morley JE, Lucia A. Exercise in people over 85. BMJ 2020;368:m402.

18 Lacroix A, Hortobágyi T, Beurskens R, et al. Effects of supervised vs. unsupervised training programs on balance and muscle strength in older adults: a systematic review and meta-analysis. Sports Med 2017;47:2341-61.

19 Izquierdo M, Casas-Herrero A, Zambm-Ferraresi F. Multicomponent physical exercise program vivifrail. A practical guide for prescribing a multicomponent physical training program to prevent weakness and falls in people over 70, 2017. Available: http://vivifrail.com/wpcontent/uploads/2019/11/VIVIFRAIL-ENG-Interactivo.pdf

20 Courel-lbáñez J, Martínez-Cava A, Morán-Navarro R, et al. Reproducibility and repeatability of five different technologies for bar velocity measurement in resistance training. Ann Biomed Eng 2019;47:1523-38.

21 Martínez-Cava A, Hernández-Belmonte A, Courel-lbáñez J. Effect of pause vs. rebound techniques on neuromuscular and functional performance after a prolonged velocity-based training. Int J Sports Physiol Perform 2021.

22 Pallarés JG, Cava AM, Courel-Ibáñez J, et al. Full squat produces greater neuromuscular and functional adaptations and lower pain than partial squats after prolonged resistance training. Eur J Sport Sci 2020;20:115-24.

23 Pareja-Blanco F, Alcazar J, Cornejo-Daza PJ, et al. Effects of velocity loss in the bench press exercise on strength gains, neuromuscular adaptations, and muscle hypertrophy. Scand J Med Sci Sports 2020;30:2154-66.

24 Martínez-Cava A, Hernández-Belmonte A, Courel-lbáñez J, et al. Bench press at full range of motion produces greater neuromuscular adaptations than partial executions after prolonged resistance training. J Strength Cond Res 2019

25 Rodríguez-Rielves V, Martínez-Cava A, Buendía-Romero Ángel, et al. Reproducibility of the rotor 2INpower Crankset for monitoring cycling power output: a comprehensive analysis in different Real-Context situations. Int J Sports Physiol Perform 2021;1:1-6.

26 Mujika I, Padilla S. Detraining: loss of training-induced physiological and performance adaptations. Part I. Sports Med 2000;30:79-87.

27 Gill TM, Gahbauer EA, Han L, et al. The relationship between intervening hospitalizations and transitions between frailty states. J Gerontol A Biol Sci Med Sci 2011;66:1238-43.

28 Blocquiaux S, Gorski T, Van Roie E, et al. The effect of resistance training, detraining and retraining on muscle strength and power, myofibre size, satellite cells and myonuclei in older men. Exp Gerontol 2020;133:110860.

29 Toraman NF, Ayceman N. Effects of six weeks of detraining on retention of functional fitness of old people after nine weeks of multicomponent training. Br J Sports Med 2005;39:565-8.

30 Blasco-Lafarga C, Cordellat A, Forte A, et al. Short and long-term trainability in older adults: training and detraining following two years of multicomponent cognitive-physical exercise training. Int $J$ Environ Res Public Health 2020;17:5984-16.

31 Toraman NF. Short term and long term detraining: is there any difference between young-old and old people? Br J Sports Med 2005;39:561-4.

32 Leitão L, Pereira A, Mazini M, et al. Effects of three months of detraining on the health profile of older women after a multicomponent exercise program. Int J Environ Res Public Health 2019;16:3881-11.

33 Cadore EL, Moneo ABB, Mensat MM, et al. Positive effects of resistance training in frail elderly patients with dementia after longterm physical restraint. Age 2014;36:801-11. 
34 Bosquet L, Berryman N, Dupuy O, et al. Effect of training cessation on muscular performance: a meta-analysis. Scand J Med Sci Sports 2013;23:e140-9.

35 Bosquet L, Berryman N, Dupuy O, et al. Effect of training cessation on muscular performance: a meta-analysis. Scand J Med Sci Sports 2013;23:e140-9.
36 Van Ancum JM, Scheerman K, Jonkman NH, et al. Change in muscle strength and muscle mass in older hospitalized patients: a systematic review and meta-analysis. Exp Gerontol 2017;92:34-41.

37 Page MJ, McKenzie JE, Bossuyt PM, et al. The PRISMA 2020 statement: an updated guideline for reporting systematic reviews. Syst Rev 2021;10. 